\title{
Title: Modeling inter-continental transport of ozone in North America with CAMx for the Air Quality Model Evaluation International Initiative (AQMEII) Phase 3
}

Authors: Uarporn Nopmongcol ${ }^{1}$, Zhen Liu ${ }^{1}$, Till Stoeckenius ${ }^{1}$, Greg Yarwood $^{1}$

${ }^{1}$ Ramboll Environ, 773 San Marin Dr., Suite 2115, Novato, CA 94945

Corresponding author: Uarporn Nopmongcol (unopmongcol@ramboll.com)

Keywords: Ozone, US Emissions, Global Emissions, boundary conditions, tracer 
Table S1: Definition of WRF 35 vertical levels and mapping to the 26 vertical layers used in CAMx. Heights (m) are geopotential heights above ground level. RTCMC aggregates $\mathrm{O}_{3}$ boundary contributions from layers 1 to 16 (O3A; O3D), 17-23 (O3B; O3E), and 24-26 (O3C; O3F)

\begin{tabular}{|c|c|c|c|c|c|c|c|c|}
\hline \multirow[b]{2}{*}{ Layer } & \multicolumn{2}{|c|}{ WRF } & \multirow[b]{2}{*}{ Depth (m) } & \multicolumn{4}{|c|}{$\mathbf{C A M x}$} & \\
\hline & Pressure mb) & Height (m) & & Layer & Pressure (mb) & Height (m) & Depth (m) & \\
\hline 35 & 50 & 19260.0 & 3397 & & & & & \\
\hline 34 & 97.5 & 15863.2 & 2233 & 26 & 97.5 & 15863 & 2233 & $\begin{array}{l}\text { O3C, } \\
\text { O3F }\end{array}$ \\
\hline 33 & 145 & 13630.3 & 1701 & 25 & 145 & 13630 & 1701 & \\
\hline 32 & 192.5 & 11929.7 & 1389 & 24 & 192.5 & 11930 & 1389 & \\
\hline 31 & 240 & 10541.2 & 1181 & 23 & 240 & 10541 & 1181 & $\begin{array}{l}\text { O3B, } \\
\text { O3E }\end{array}$ \\
\hline 30 & 287.5 & 9360.1 & 1032 & 22 & 287.5 & 9360 & 1032 & \\
\hline 29 & 335 & 8327.8 & 920 & 21 & 335 & 8328 & 920 & \\
\hline 28 & 382.5 & 7407.9 & 832 & 20 & 382.5 & 7408 & 1592 & \\
\hline 27 & 430 & 6576.3 & 760 & & & & & \\
\hline 26 & 477.5 & 5816.1 & 701 & 19 & 477.5 & 5816 & 1353 & \\
\hline 25 & 525 & 5114.9 & 652 & & & & & \\
\hline 24 & 572.5 & 4463.3 & 609 & 18 & 572.5 & 4463 & 1182 & \\
\hline 23 & 620 & 3854.1 & 573 & & & & & \\
\hline 22 & 667.5 & 3281.6 & 540 & 17 & 667.5 & 3282 & 952 & \\
\hline 21 & 715 & 2741.1 & 412 & & & & & \\
\hline 20 & 753 & 2329.4 & 298 & 16 & 753 & 2329 & 587 & $\begin{array}{l}\text { O3A, } \\
\text { O3D }\end{array}$ \\
\hline 19 & 781.5 & 2031.4 & 289 & & & & & \\
\hline 18 & 810 & 1742.2 & 188 & 15 & 810 & 1742 & 373 & \\
\hline 17 & 829 & 1553.9 & 185 & & & & & \\
\hline 16 & 848 & 1369.1 & 182 & 14 & 848 & 1369 & 360 & \\
\hline 15 & 867 & 1187.5 & 178 & & & & & \\
\hline 14 & 886 & 1009.2 & 175 & 13 & 886 & 1009 & 175 & \\
\hline 13 & 905 & 834.0 & 87 & 12 & 905 & 834 & 87 & \\
\hline 12 & 914.5 & 747.5 & 86 & 11 & 914.5 & 747 & 171 & \\
\hline 11 & 924 & 661.7 & 85 & & & & & \\
\hline 10 & 933.5 & 576.6 & 84 & 10 & 933.5 & 577 & 84 & \\
\hline 9 & 943 & 492.3 & 84 & 9 & 943 & 492 & 84 & \\
\hline 8 & 952.5 & 408.6 & 83 & 8 & 952.5 & 409 & 83 & \\
\hline 7 & 962 & 325.6 & 82 & 7 & 962 & 326 & 82 & \\
\hline 6 & 971.5 & 243.2 & 82 & 6 & 971.5 & 243 & 82 & \\
\hline 5 & 981 & 161.5 & 41 & 5 & 981 & 162 & 41 & \\
\hline 4 & 985.75 & 120.9 & 40 & 4 & 985.75 & 121 & 40 & \\
\hline 3 & 990.5 & 80.4 & 40 & 3 & 990.5 & 80 & 40 & \\
\hline 2 & 995.25 & 40.1 & 20 & 2 & 995.25 & 40 & 20 & \\
\hline 1 & 997.625 & 20.1 & 20 & 1 & 997.625 & 20 & 20 & \\
\hline 0 & 1000 & 0.0 & & 0 & 1000 & 0 & 0 & \\
\hline
\end{tabular}


Table S2: RTCMC input file for an active (A) and inert (D) tracer groups. Here tracer group A tracks boundary contributions from the lowest 16 layers. HOXA and O1DA are additional species added to track chemical destruction of O3A. An inert tracer O3D needs to be listed in the input file even though it does not undergo any chemical reaction. Other active (B,C) and inert $(\mathrm{E}, \mathrm{F})$ tracer groups have similar expressions.

\#Equation ; Rate constants from CB05

\begin{tabular}{|c|c|}
\hline $001[03 A]->$ [O1DA] & ; $0 \quad 0.000 E+00 \quad 0.0000 E+00$ \\
\hline $002[01 D A]->[03 A]$ & ; 5 1.260E-09 1.0200E+02 \\
\hline 003 [01DA] -> & ; 15 1.320E-08 0.0000E+00 \\
\hline $004[\mathrm{O} 3 \mathrm{~A}]+[\mathrm{OH}] \quad->[\mathrm{HOXA}]$ & ; 2 1.020E-10 -9.4000E+02 \\
\hline $005[\mathrm{O} 3 \mathrm{~A}]+[\mathrm{HO} 2] \quad->[\mathrm{HOXA}]$ & ; 2 6.000E-13 -4.9000E+02 \\
\hline $006[\mathrm{HOXA}]+[\mathrm{NO}] \quad->[\mathrm{O} 3 \mathrm{~A}]$ & ; 2 2.100E-10 2.5000E+02 \\
\hline $007[\mathrm{HOXA}]+[\mathrm{HO} 2] \quad->$ & ; 19 1.380E-11 6.00E+02 1.020E-31 1.00E+03 \\
\hline $008[\mathrm{HOXA}]+[\mathrm{HO} 2] \quad->$ & ; 20 1.932E-32 2.80E+03 1.428E-52 3.20E+03 \\
\hline $009[\mathrm{HOXA}]+[\mathrm{MEO} 2] \quad->$ & ; 2 2.460E-11 7.50E+02 \\
\hline $010[\mathrm{HOXA}]+[\mathrm{XO} 2] \quad->$ & ; 2 4.500E-11 7.0000E+02 \\
\hline $011[\mathrm{HOXA}]+[\mathrm{XO} 2 \mathrm{~N}] \quad->$ & ; 2 4.500E-11 7.0000E+02 \\
\hline $012[\mathrm{HOXA}]+[\mathrm{C2O}] \quad->(0.2)[03 \mathrm{~A}]$ & ; $22.58 \mathrm{E}-111.0400 \mathrm{E}+03$ \\
\hline $013[\mathrm{HOXA}]+[\mathrm{CXO}]-\rightarrow(0.2)[\mathrm{O} 3 \mathrm{~A}]$ & ; 2 2.58E-11 1.0400E+03 \\
\hline $014[\mathrm{O} 3 \mathrm{~A}]+[\mathrm{ISOP}]->$ & ; 2 4.716E-13 -1.9120E+03 \\
\hline $015[\mathrm{O} 3 \mathrm{~A}]+[\mathrm{TERP}] \quad->$ & ; 2 7.2E-14 -8.2100E+02 \\
\hline $046[03 D] \quad->$ & ; 10.0 \\
\hline
\end{tabular}

Table S3: Model performance statistics for MDA8 $\mathrm{O}_{3}$ for CAMx basecase (no cutoff).

\begin{tabular}{|c|c|c|c|c|c|c|c|c|c|}
\hline Major City & AQS ID & \#Obs & $\begin{array}{l}\text { Model } \\
\text { Mean } \\
\text { (ppb) }\end{array}$ & $\begin{array}{c}\text { NMB } \\
(\%)\end{array}$ & $\begin{array}{c}\text { NME } \\
(\%)\end{array}$ & $\begin{array}{c}\text { FB } \\
(\%)\end{array}$ & $\begin{array}{c}\text { FE } \\
(\%)\end{array}$ & $r$ & RMSE \\
\hline Atlanta, GA & 131210055 & 242 & 52.7 & 4.2 & 17.6 & 4.7 & 18.3 & 0.71 & 11.15 \\
\hline Birmingham, AL & 10732006 & 232 & 51.7 & 5.2 & 18.1 & 6.4 & 18.9 & 0.63 & 11.27 \\
\hline Boise, ID & 160010017 & 166 & 48.1 & -6.0 & 11.6 & -5.7 & 11.9 & 0.60 & 7.50 \\
\hline Boston, MA & 440090007 & 208 & 50.3 & 7.8 & 15.1 & 8.3 & 15.3 & 0.76 & 8.86 \\
\hline Chicago, IL & 550590019 & 220 & 47.3 & -2.8 & 13.1 & -2.5 & 13.5 & 0.78 & 8.41 \\
\hline Cincinnati, $\mathrm{OH}$ & 390610006 & 212 & 52.9 & 1.0 & 13.1 & 1.2 & 14.1 & 0.77 & 9.34 \\
\hline Columbus, $\mathrm{OH}$ & 390490029 & 211 & 51.3 & -1.7 & 12.5 & -1.5 & 13.7 & 0.76 & 8.47 \\
\hline Dallas, TX & 484392003 & 354 & 42.8 & -3.0 & 17.0 & -3.9 & 18.7 & 0.80 & 9.24 \\
\hline Denver, CO & 80350004 & 344 & 48.7 & -0.4 & 14.3 & 0.7 & 15.1 & 0.68 & 9.47 \\
\hline Detroit, MI & 261630019 & 167 & 47.1 & -5.4 & 14.3 & -4.5 & 15.2 & 0.76 & 8.93 \\
\hline Houston, TX & 480391004 & 353 & 45.2 & 7.9 & 21.1 & 10.4 & 21.4 & 0.68 & 12.17 \\
\hline Kansas City, KS & 290490001 & 214 & 51.3 & 4.3 & 13.7 & 5.0 & 13.7 & 0.74 & 8.51 \\
\hline $\begin{array}{l}\text { Los Angeles, CA } \\
\text { Minneapolis, }\end{array}$ & 60710005 & 358 & 56.6 & -3.3 & 12.5 & -3.5 & 12.9 & 0.88 & 9.65 \\
\hline $\mathrm{MN}$ & 270031002 & 339 & 35.2 & -8.3 & 20.6 & -11 & 23.7 & 0.67 & 9.75 \\
\hline New York, NY & 340290006 & 257 & 51.6 & 6.5 & 15.2 & 7.9 & 16.3 & 0.82 & 9.65 \\
\hline Philadelphia, PA & 421010024 & 362 & 43.4 & -2.5 & 14.4 & -2.7 & 16 & 0.88 & 8.16 \\
\hline Phoenix, AZ & 40131004 & 363 & 43.9 & -11.9 & 15.2 & -12.6 & 16.7 & 0.84 & 9.97 \\
\hline Pittsburgh, PA & 420031005 & 356 & 46.3 & 8.8 & 16.2 & 9.0 & 16.8 & 0.85 & 9.15 \\
\hline Sacramento, CA & 60670012 & 338 & 46.0 & -0.6 & 13.1 & 2.8 & 15.2 & 0.88 & 8.16 \\
\hline Salt Lake, UT & 490110004 & 152 & 52.3 & 3.0 & 12.7 & 3.5 & 12.6 & 0.56 & 8.46 \\
\hline
\end{tabular}




\begin{tabular}{llllllllll} 
St. Louis, MO & 291831002 & 214 & 53.4 & 2.7 & 12.9 & 3.5 & 12.8 & 0.80 & 8.40 \\
Baltimore, MD & 240251001 & 189 & 57.9 & 1.8 & 12.9 & 3.5 & 14.3 & 0.86 & 9.37 \\
\hline
\end{tabular}

Table S4: Model performance statistics for $\mathrm{MDA8}_{3}$ with 40 ppb cutoff for CAMx basecase

\begin{tabular}{|c|c|c|c|c|c|c|c|c|c|}
\hline Major City & AQS ID & \#Obs & $\begin{array}{l}\text { Model } \\
\text { Mean } \\
\text { (ppb) }\end{array}$ & $\begin{array}{c}\text { NMB } \\
(\%)\end{array}$ & $\begin{array}{c}\text { NME } \\
(\%)\end{array}$ & $\begin{array}{c}\text { FB } \\
(\%)\end{array}$ & $\begin{array}{c}\text { FE } \\
(\%)\end{array}$ & $r$ & RMSE \\
\hline Atlanta, GA & 131210055 & 184 & 56.8 & 0.44 & 15.4 & -0.8 & 15.8 & 0.60 & 11.08 \\
\hline Birmingham, AL & 10732006 & 172 & 55.0 & 0.05 & 14.77 & -0.4 & 15.1 & 0.48 & 10.67 \\
\hline Boise, ID & 160010017 & 147 & 49.02 & -7.53 & 11.54 & -7.7 & 11.9 & 0.48 & 7.69 \\
\hline Boston, MA & 440090007 & 148 & 53.7 & 3.48 & 12.57 & 2.8 & 12.5 & 0.70 & 8.39 \\
\hline Chicago, IL & 550590019 & 159 & 51.0 & -5.68 & 13.16 & -6.6 & 13.9 & 0.71 & 9.21 \\
\hline Cincinnati, $\mathrm{OH}$ & 390610006 & 174 & 56.1 & -1.31 & 11.38 & -2.2 & 11.9 & 0.74 & 8.50 \\
\hline Columbus, $\mathrm{OH}$ & 390490029 & 179 & 53.6 & -3.75 & 11.03 & -4.6 & 11.8 & 0.75 & 7.94 \\
\hline Dallas, TX & 484392003 & 197 & 50.6 & -5.85 & 14.95 & -7.7 & 16.3 & 0.73 & 9.93 \\
\hline Denver, CO & 80350004 & 264 & 51.7 & -4.35 & 11.76 & -4.7 & 11.9 & 0.62 & 8.47 \\
\hline Detroit, MI & 261630019 & 131 & 50.2 & -7.85 & 13.07 & -8.2 & 13.6 & 0.69 & 8.88 \\
\hline Houston, TX & 480391004 & 183 & 51.3 & -4.03 & 13.51 & -3.9 & 13.3 & 0.66 & 10.09 \\
\hline Kansas City, KS & 290490001 & 164 & 54.3 & 1.56 & 12.26 & 1.7 & 12.1 & 0.65 & 8.38 \\
\hline $\begin{array}{l}\text { Los Angeles, CA } \\
\text { Minneapolis, }\end{array}$ & 60710005 & 300 & 60.5 & -3.92 & 12.37 & -4.7 & 12.8 & 0.86 & 10.22 \\
\hline MN & 270031002 & 143 & 43.0 & -11.48 & 17.8 & -13.9 & 20.1 & 0.36 & 11.01 \\
\hline New York, NY & 340290006 & 176 & 57.0 & 1.11 & 11.16 & 0.5 & 11.3 & 0.77 & 8.48 \\
\hline Philadelphia, PA & 421010024 & 202 & 53.5 & -4.83 & 12.17 & -6.2 & 13.3 & 0.81 & 8.75 \\
\hline Phoenix, AZ & 40131004 & 263 & 49.5 & -13.06 & 15.29 & -14.9 & 17 & 0.68 & 11.17 \\
\hline Pittsburgh, PA & 420031005 & 189 & 56.3 & 4.6 & 11.93 & 3.8 & 11.9 & 0.78 & 8.39 \\
\hline Sacramento, CA & 60670012 & 208 & 53.6 & -4.24 & 11.33 & -3.9 & 11.6 & 0.81 & 8.38 \\
\hline Salt Lake, UT & 490110004 & 134 & 53.0 & 0.69 & 11.2 & 1 & 11.1 & 0.55 & 7.57 \\
\hline St. Louis, MO & 291831002 & 176 & 56.0 & 0.64 & 11.98 & 0.8 & 11.9 & 0.74 & 8.43 \\
\hline Baltimore, MD & 240251001 & 156 & 61.7 & -1.25 & 10.68 & -1.6 & 11 & 0.83 & 8.53 \\
\hline
\end{tabular}




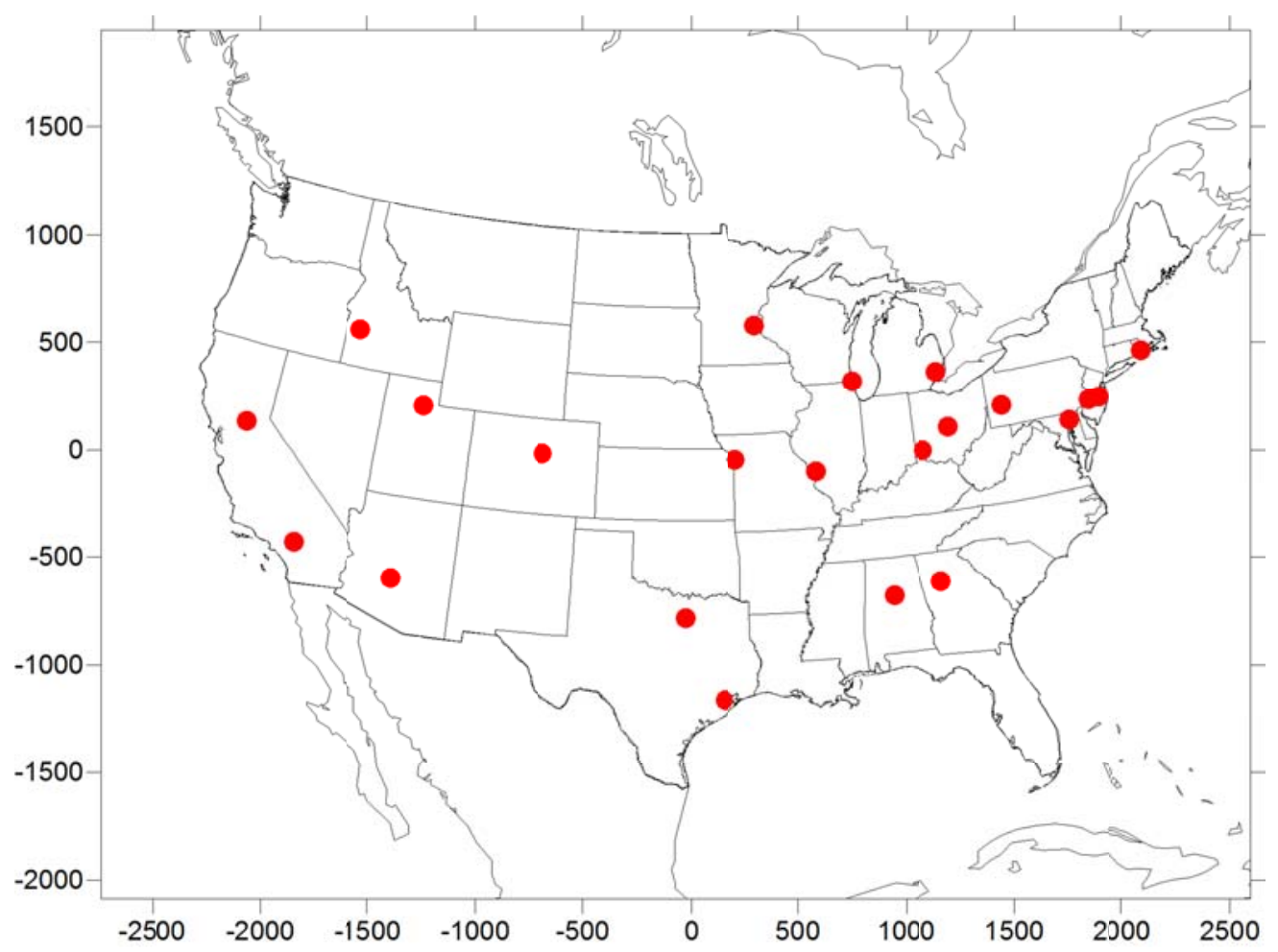

Figure S1: Circles represent 22 cities analysed in this study covering a wide variety of climatic and geographic environments. We chose their representative AQS monitoring site based on the monitoring site with highest H4MDA8 in the metropolitan statistical area.

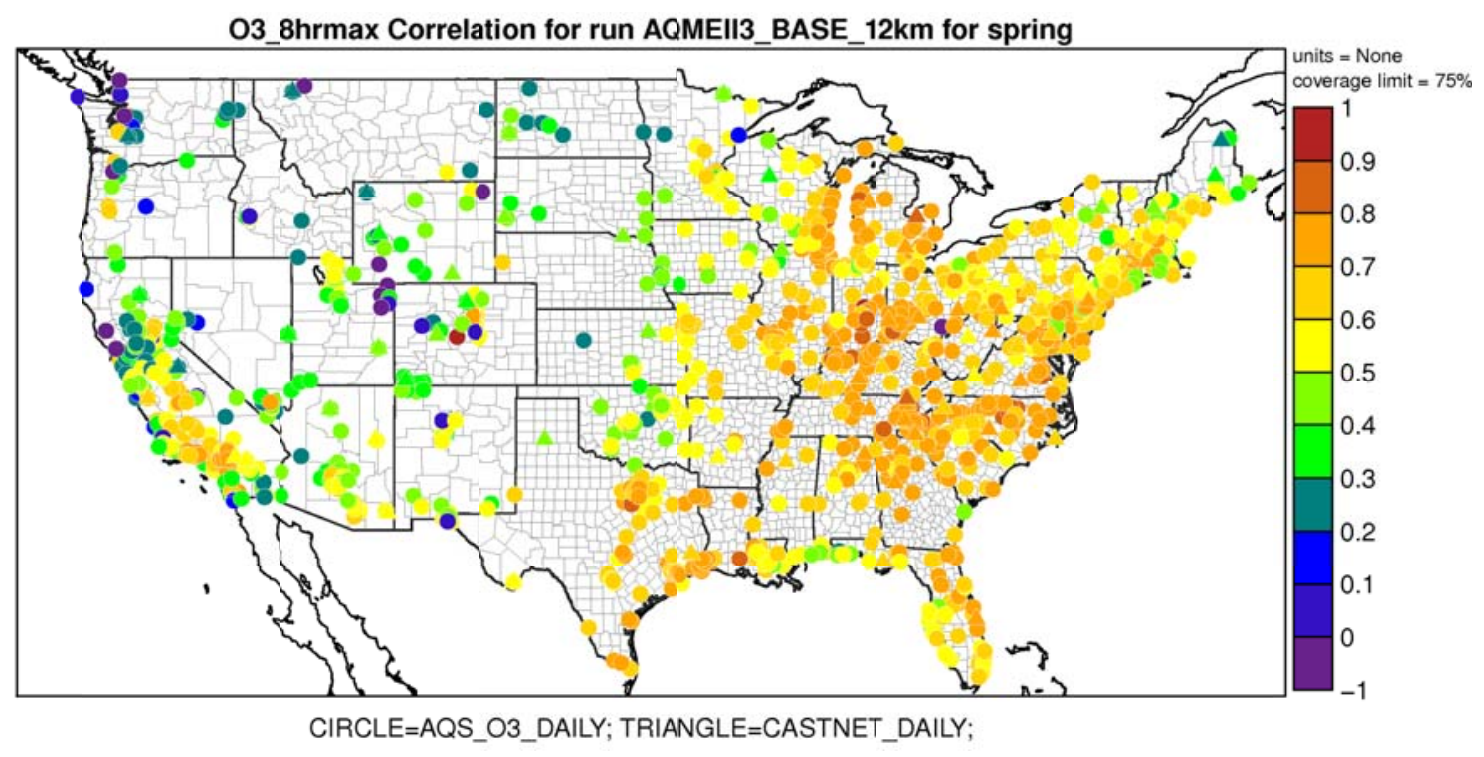

Figure S2a: Spatial map of CAMx correlation coefficient for $\mathrm{MDA8}_{3} \mathrm{O}_{3}$ with $40 \mathrm{ppb}$ cutoff in spring 
O3_8hrmax NMB (\%) for run AQMEII3_BASE_12km for spring

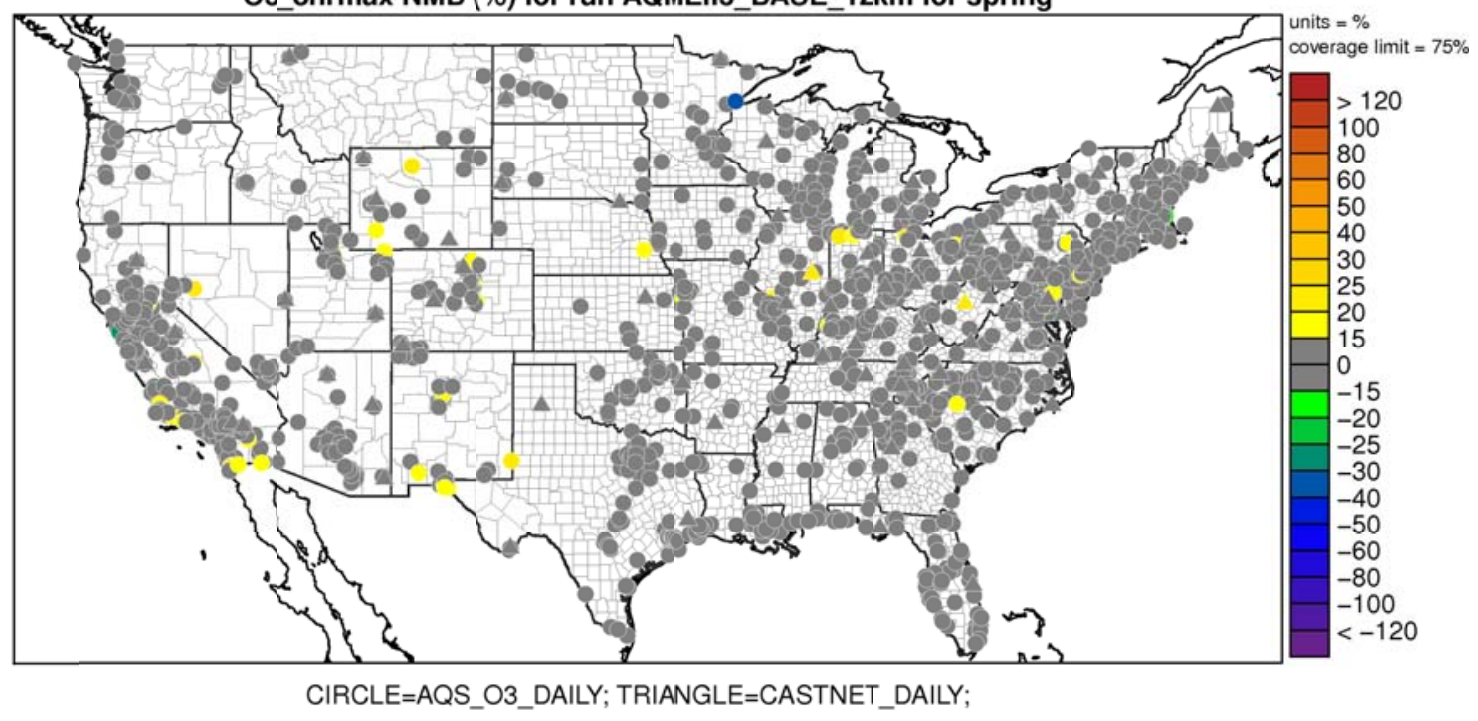

Figure S2b: Spatial map of CAMx NMB for MDA8 $\mathrm{O}_{3}$ with 40 ppb cutoff in spring 


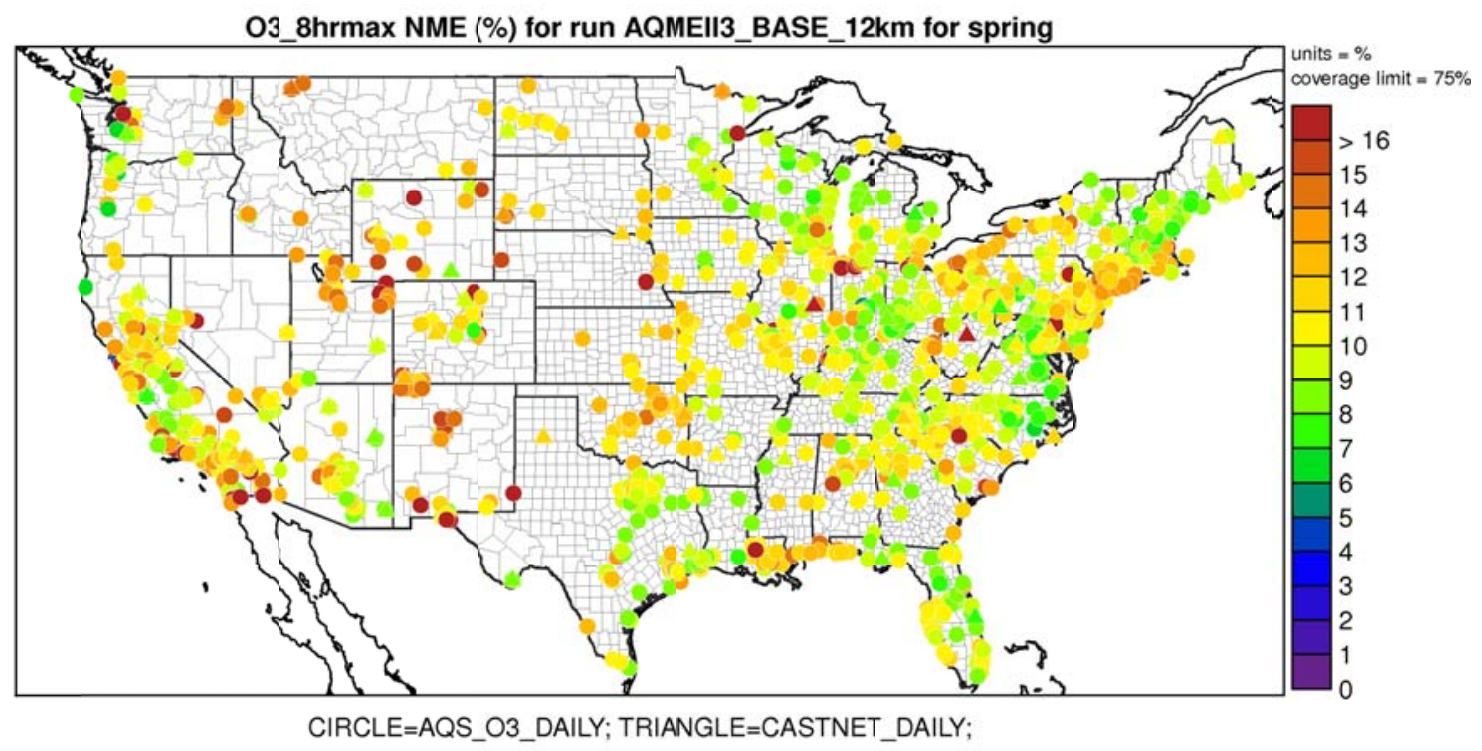

Figure S2c: Spatial map of CAMx NME for MDA8 $\mathrm{O}_{3}$ with 40 ppb cutoff in spring.

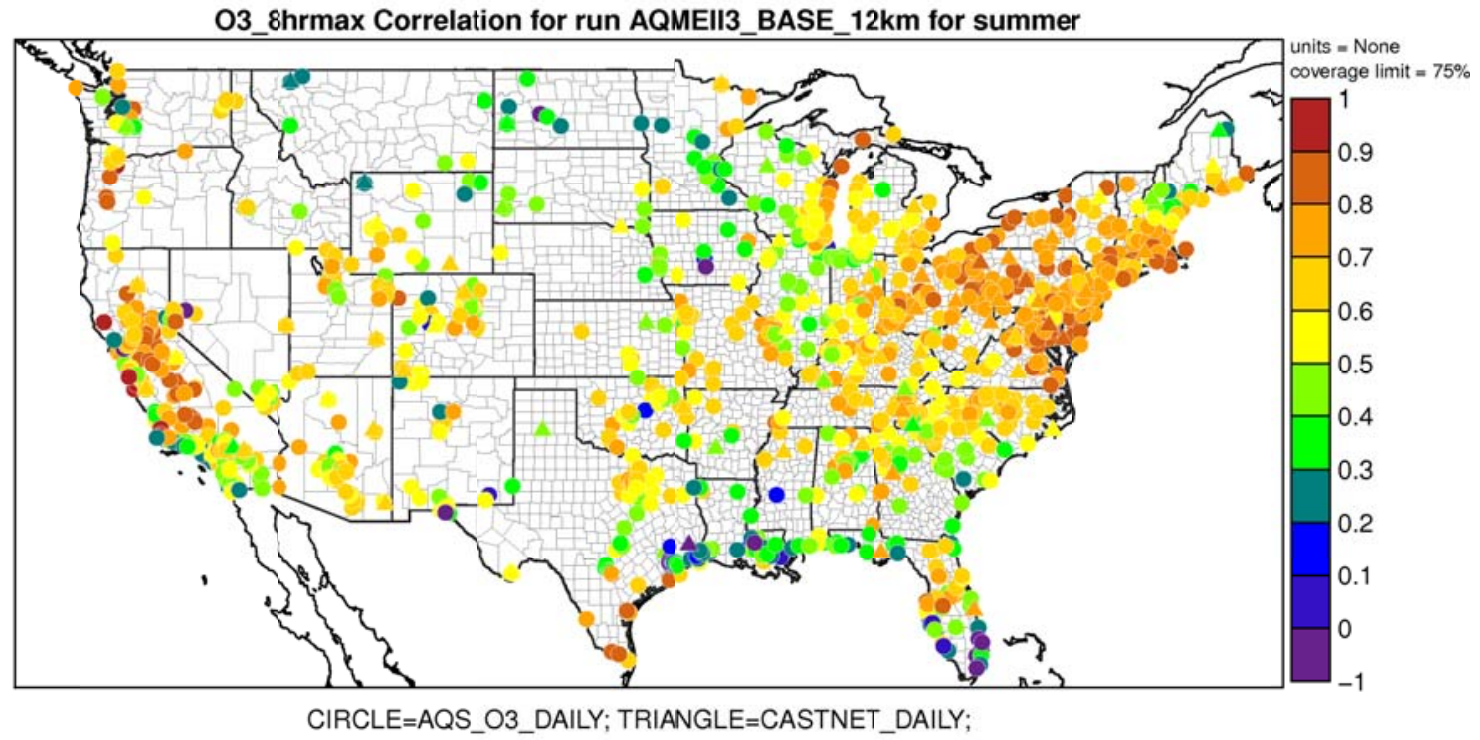

Figure S3a: Spatial map of CAMx correlation coefficnet for MDA8 $\mathrm{O}_{3}$ with 40 ppb cutoff in summer. 


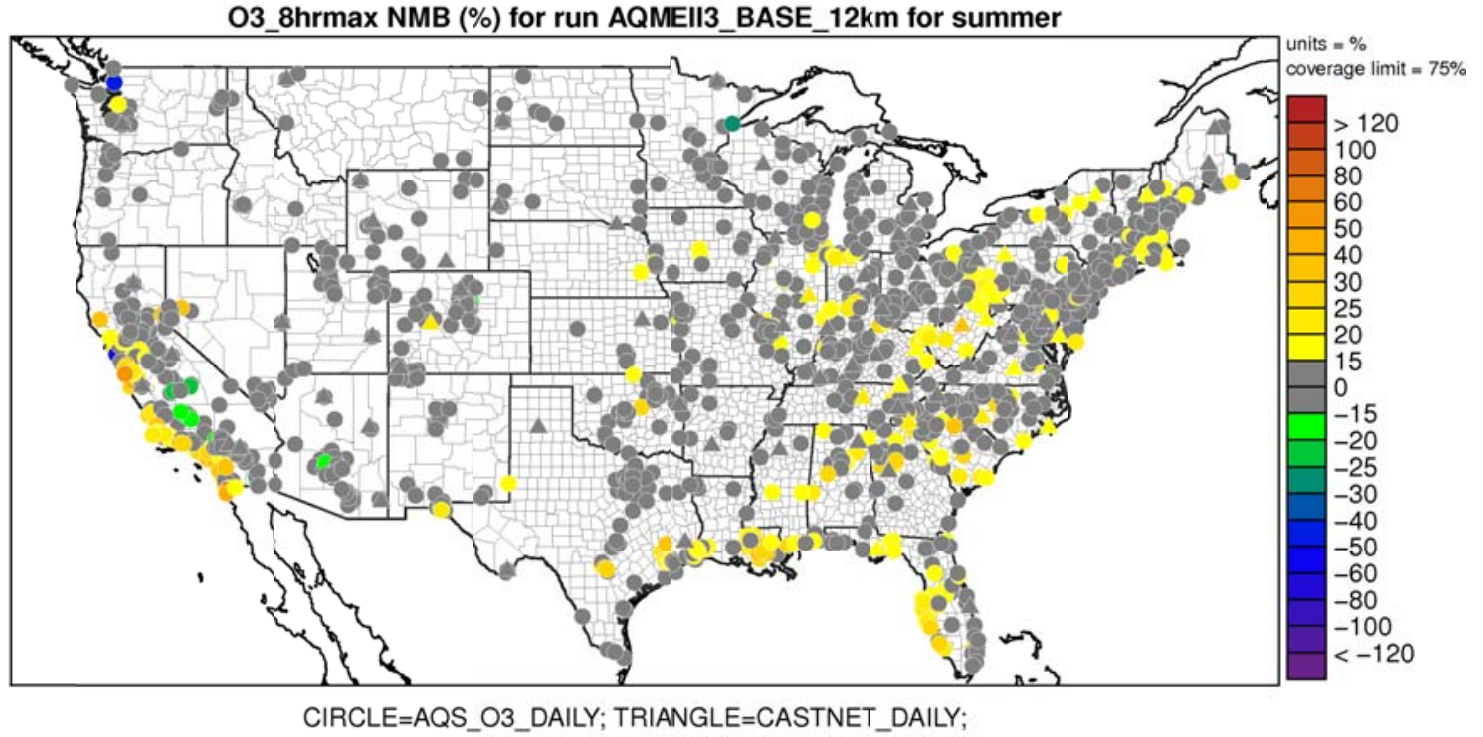

Figure S3b: Spatial map of CAMx NMB for $\mathrm{MDA8}_{3}$ with $40 \mathrm{ppb}$ cutoff in summer.

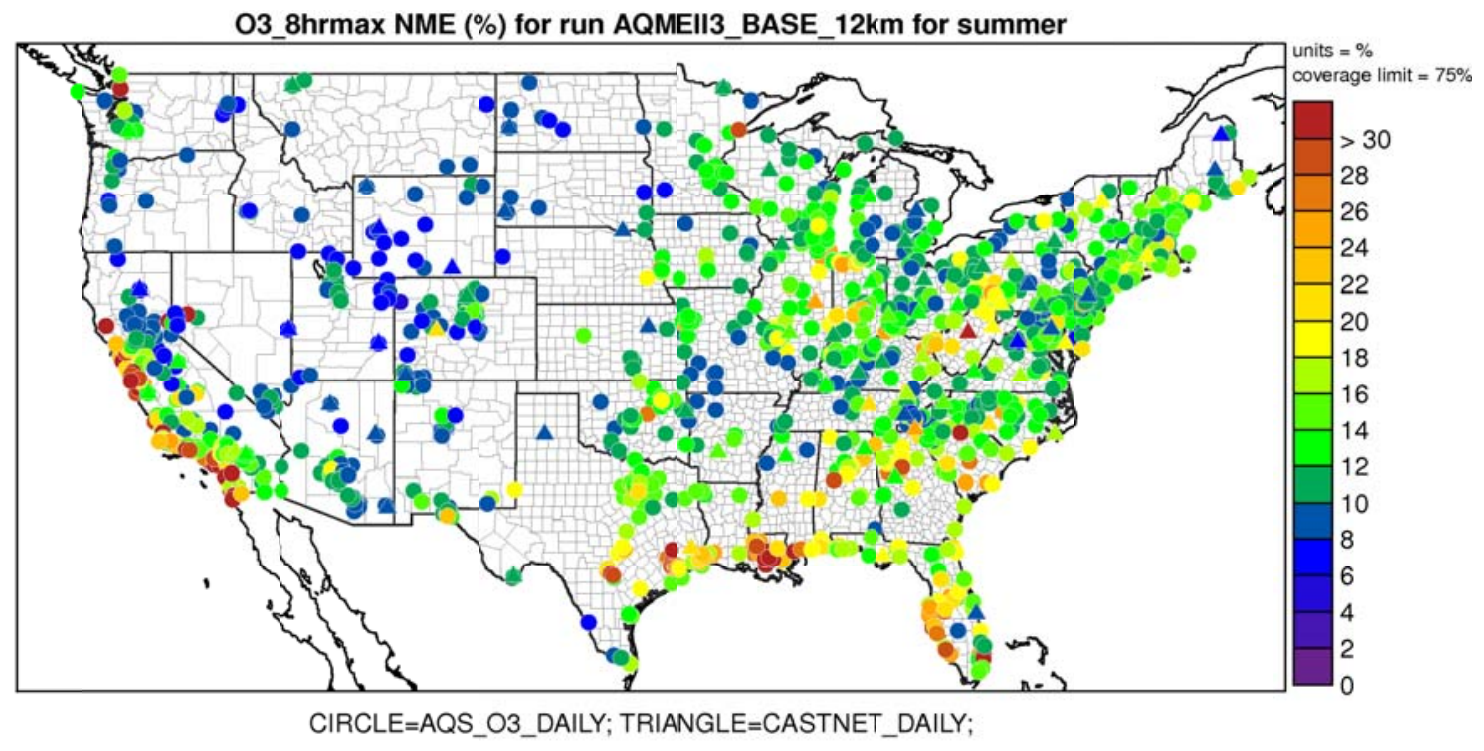

Figure S3c: Spatial map of CAMx NME for $\mathrm{MDA8}_{3}$ with $40 \mathrm{ppb}$ cutoff in summer. 


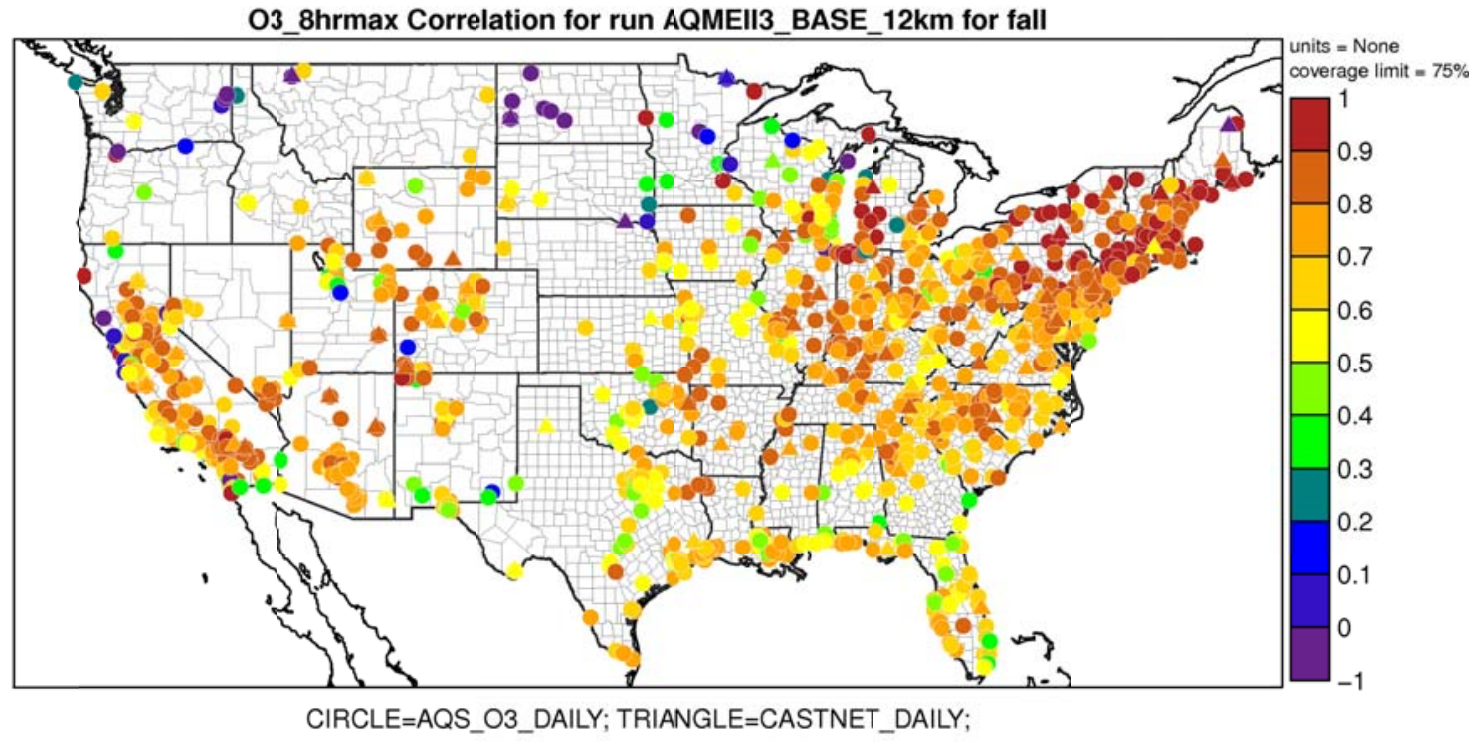

Figure S4a: Spatial map of CAMx correlation coefficient for $\mathrm{MDA8} \mathrm{O}_{3}$ with $40 \mathrm{ppb}$ cutoff in fall.

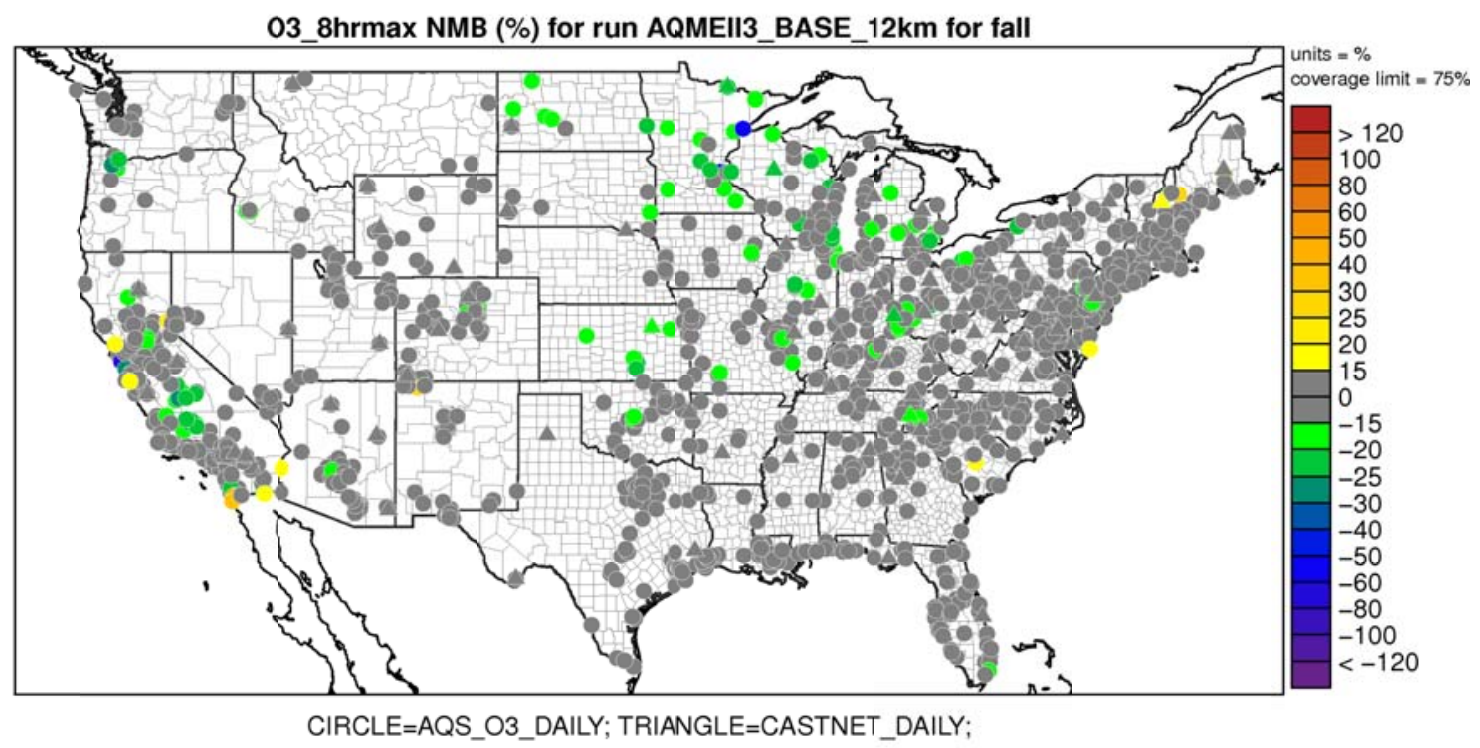

Figure S4b: Spatial map of CAMx NMB for MDA8 $\mathrm{O}_{3}$ with 40 ppb cutoff in fall. 
03_8hrmax NME (\%) for run AQMEII3_BASE_12km for fall

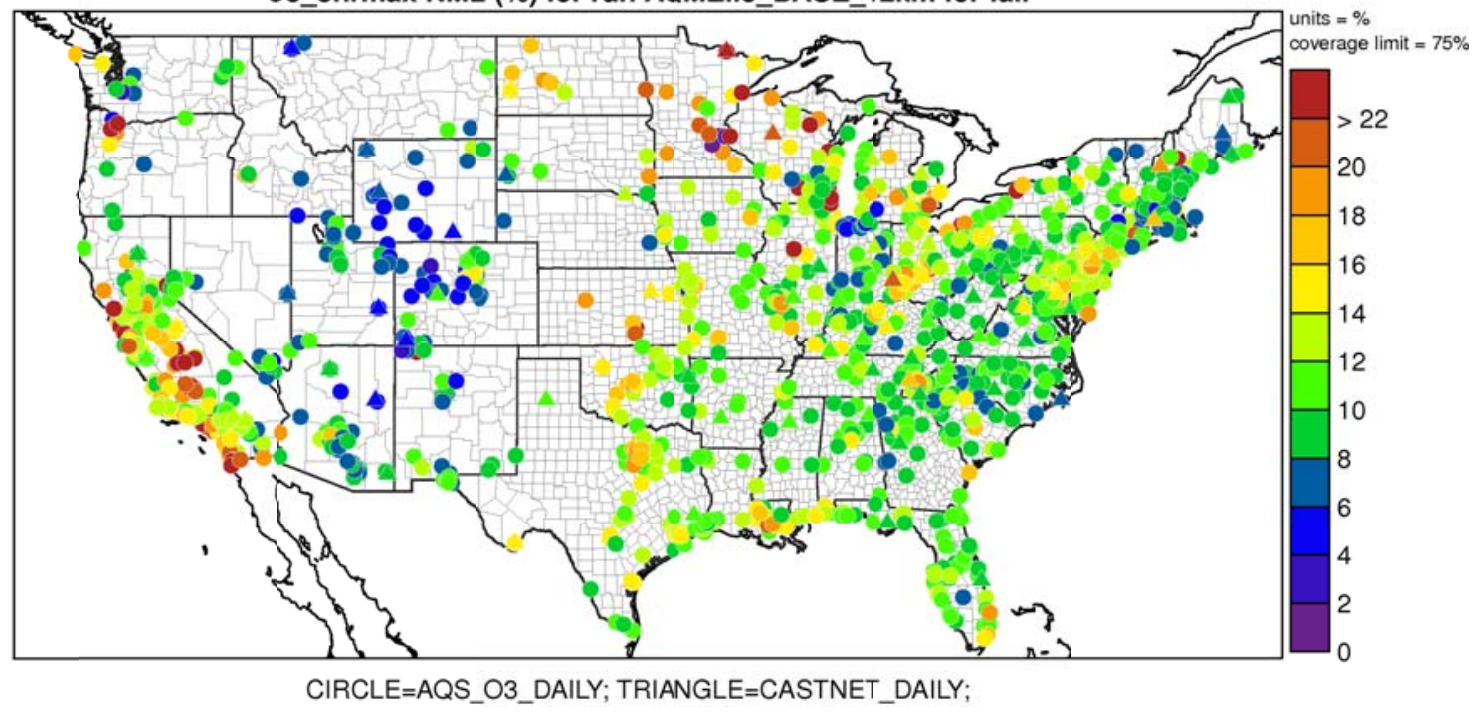

Figure S4c: Spatial map of CAMx NME for MDA8 $\mathrm{O}_{3}$ with $40 \mathrm{ppb}$ cutoff in fall.

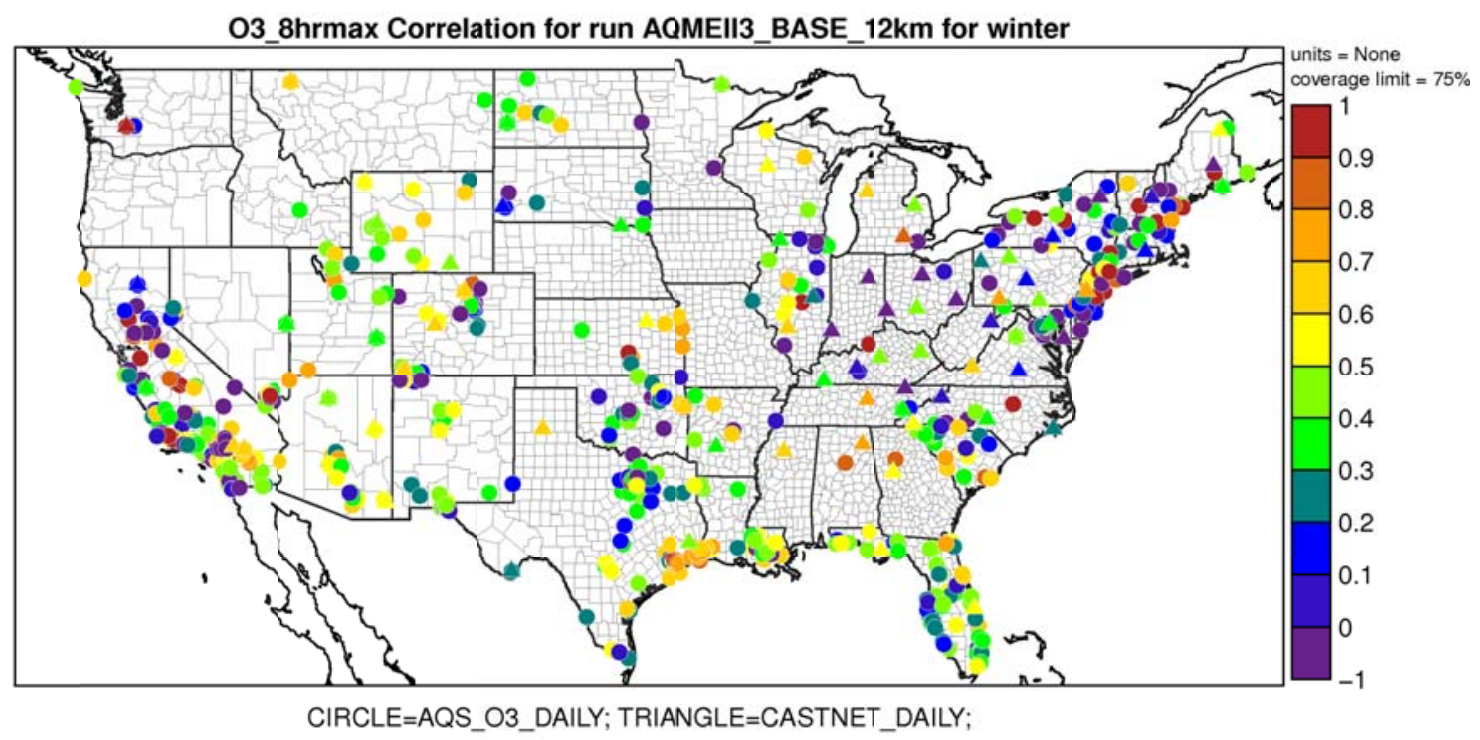

Figure S5a: Spatial map of CAMx correlation coefficient for $\mathrm{MDA8} \mathrm{O}_{3}$ with $40 \mathrm{ppb}$ cutoff in winter. 


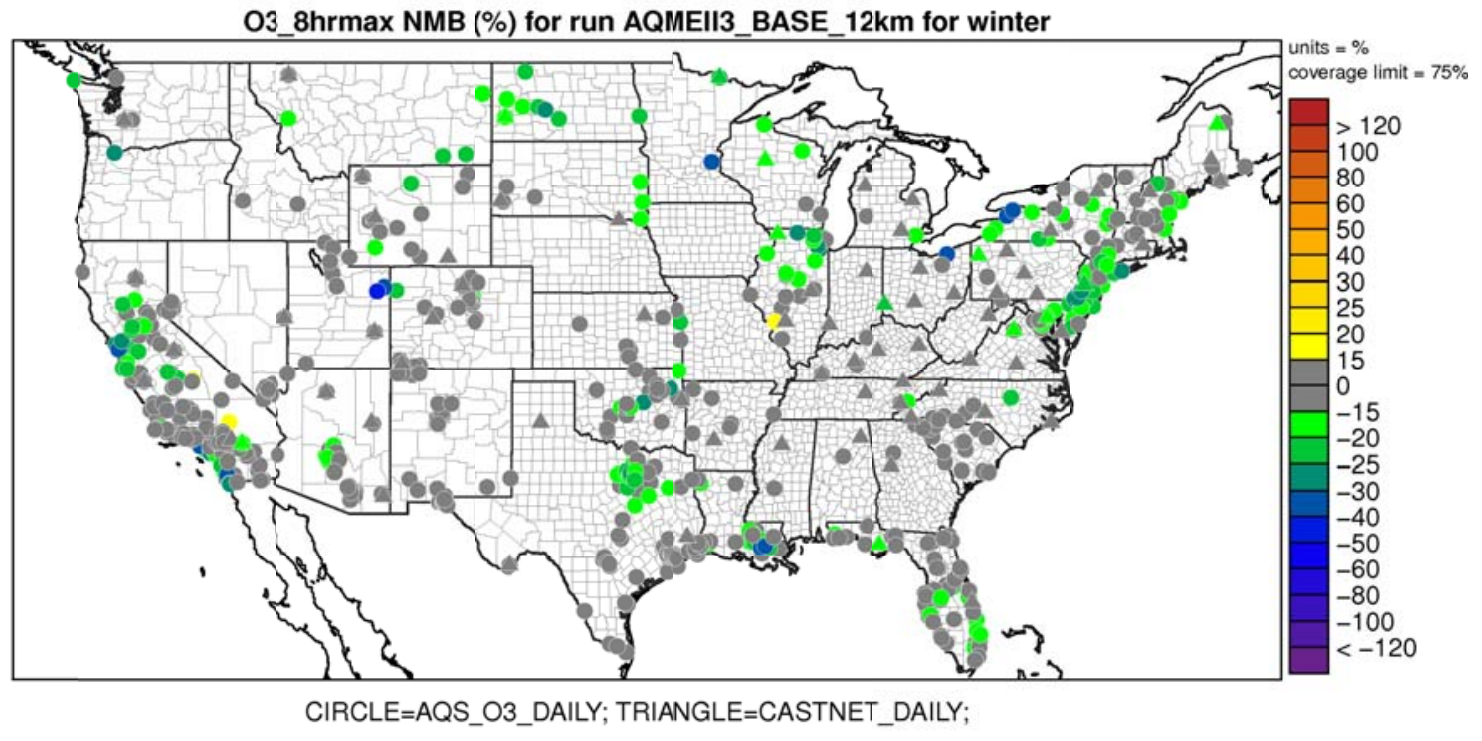

Figure S5b: Spatial map of CAMx NMB for $\mathrm{MDA8}_{3}$ with $40 \mathrm{ppb}$ cutoff in winter

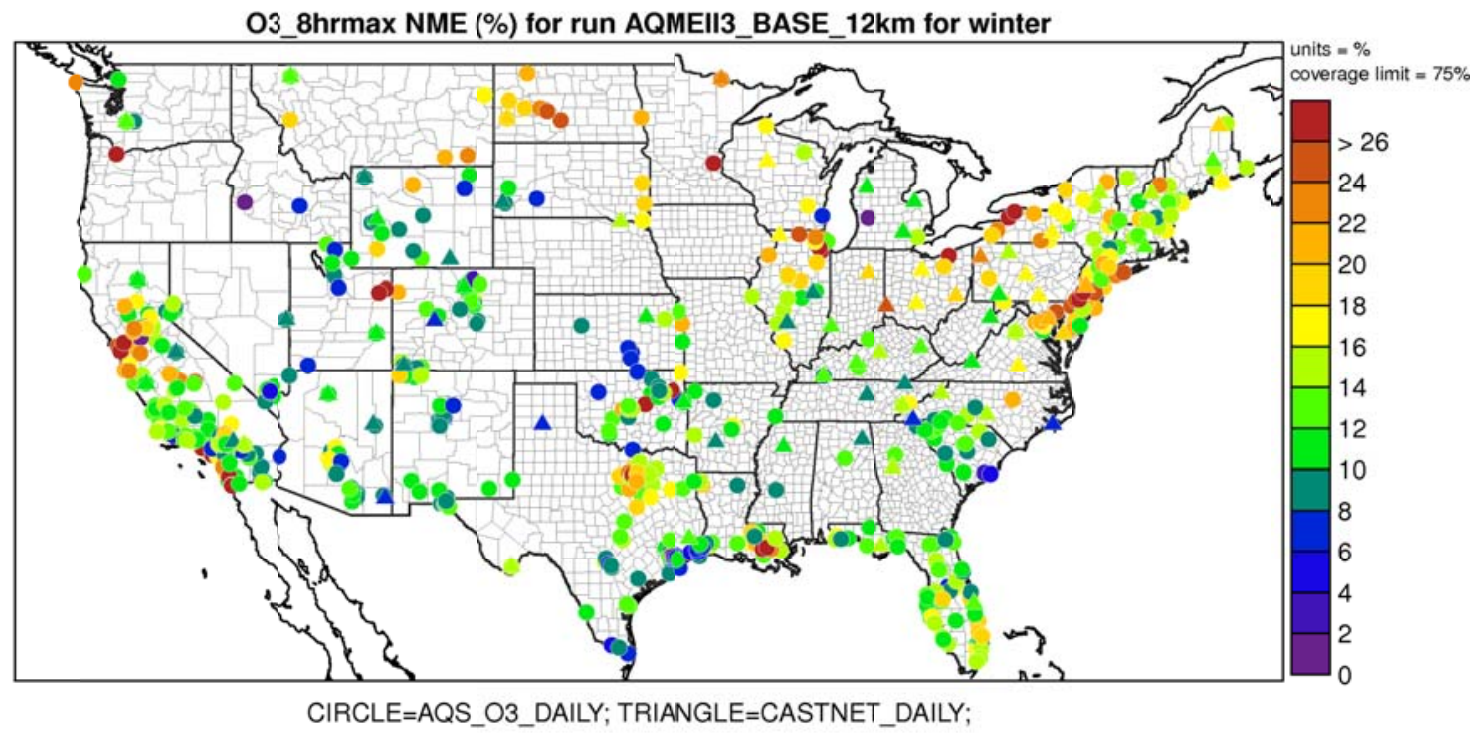

Figure S5c: Spatial map of CAMx NME for MDA8 $\mathrm{O}_{3}$ with $40 \mathrm{ppb}$ cutoff in winter 


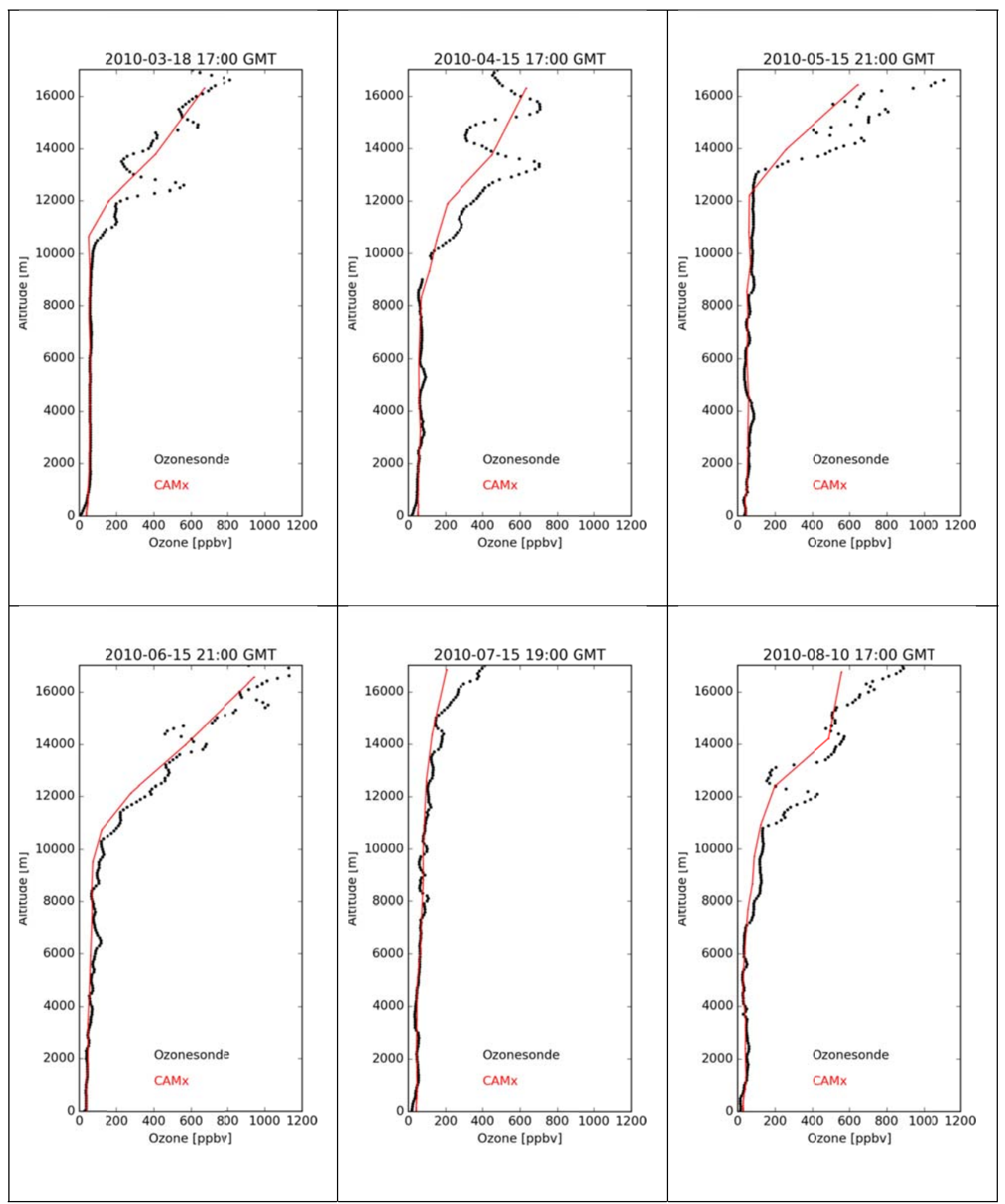

Figure S6: Vertical distribution of $\mathrm{O}_{3}$ at Trinidad Head (March-August 2010) up to 16 km. 


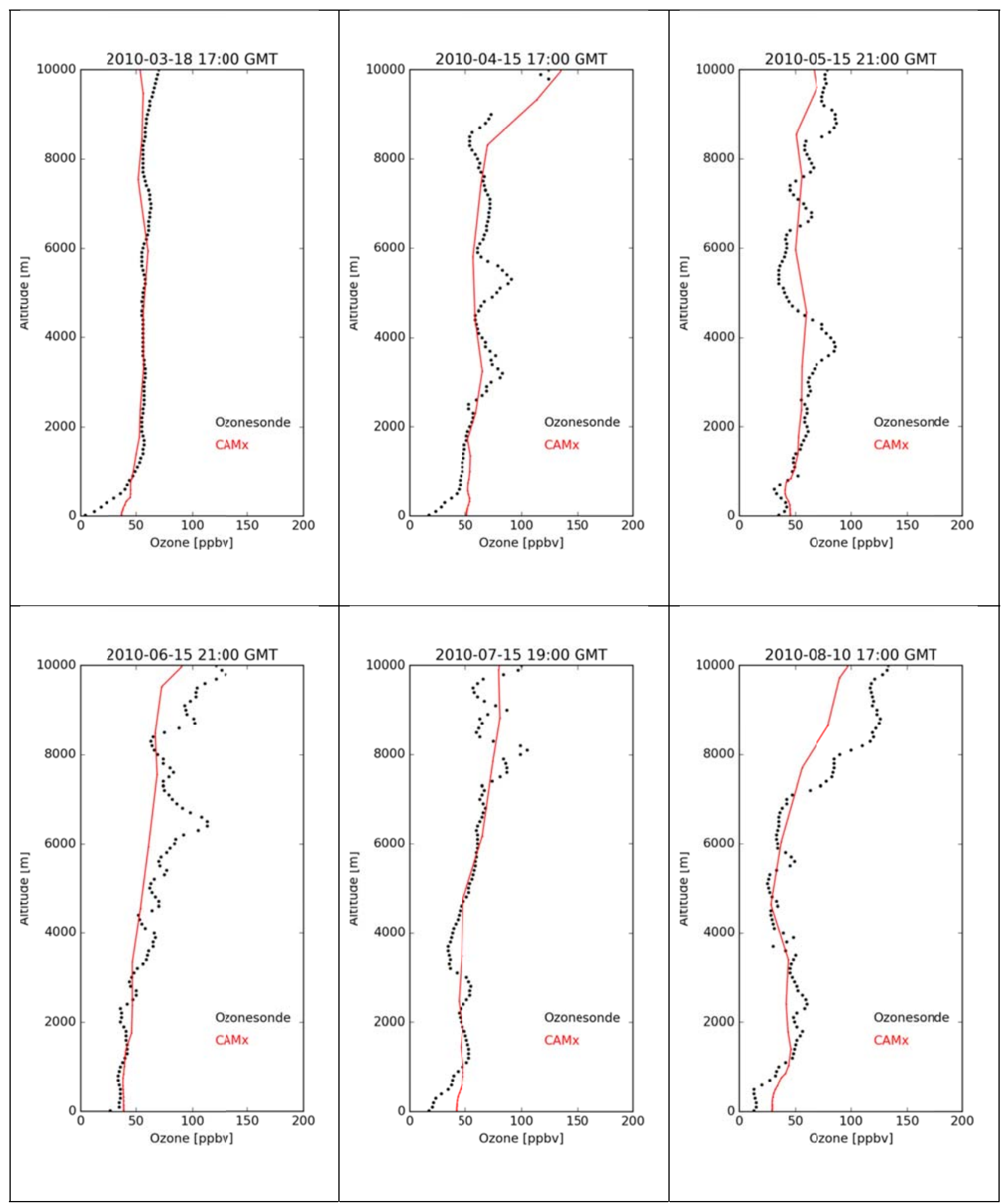

Figure S7: Same as Figure S5 but up to $10 \mathrm{~km}$. 


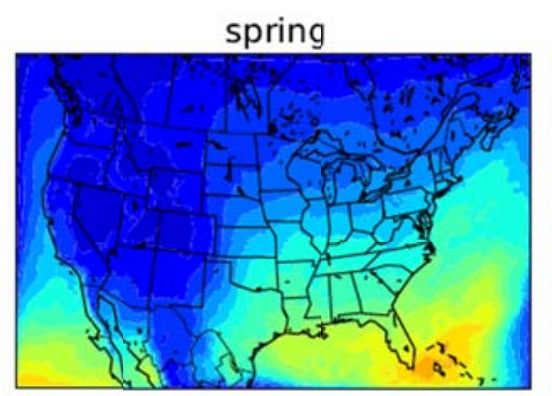

fall
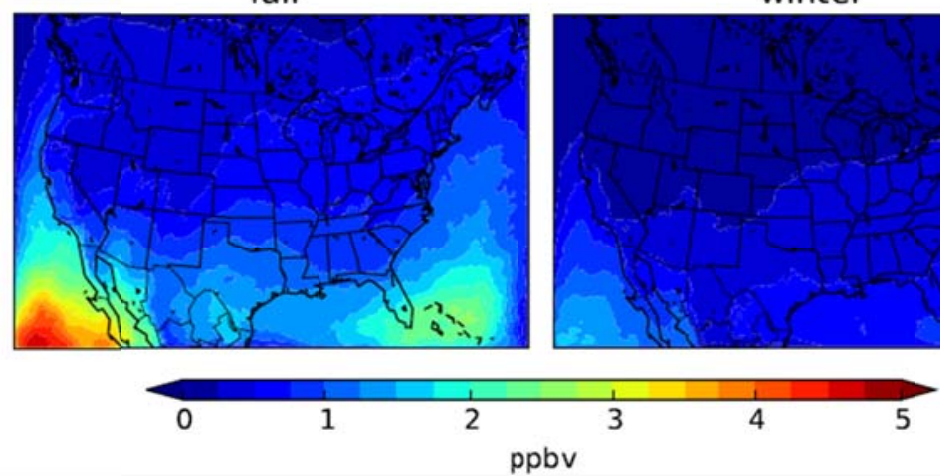

summer

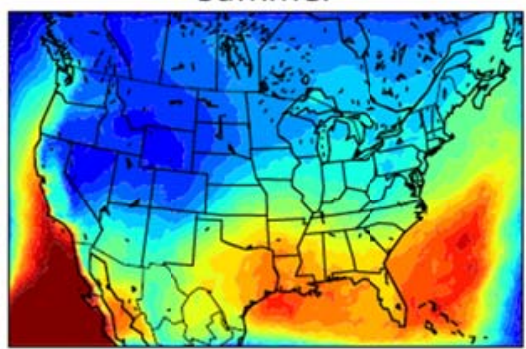

winter

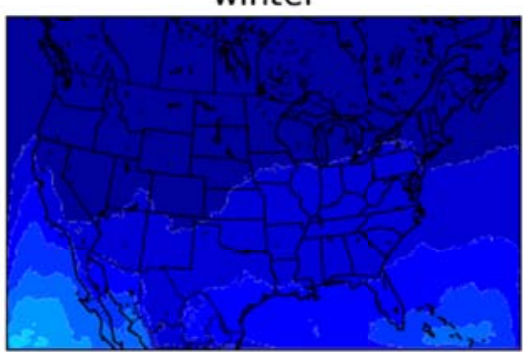

5

Figure S8: The difference in seasonal average daily maximum 8-hour $\mathrm{O}_{3}$ contribution from $\mathrm{BCs}$ inert and active $\mathrm{O}_{3}$ tracers, i.e., inert minus active, at layers below $750 \mathrm{mb}$.

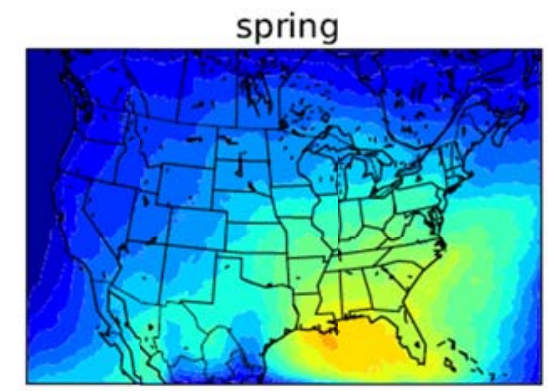

fall
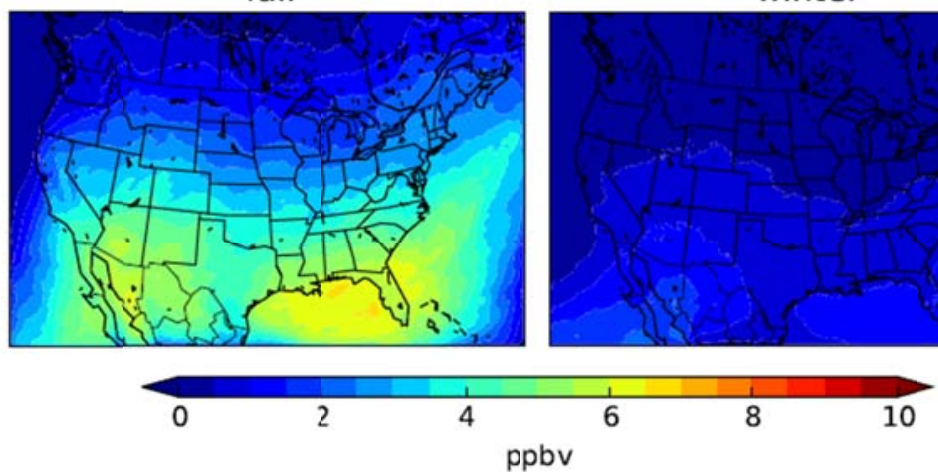

summer

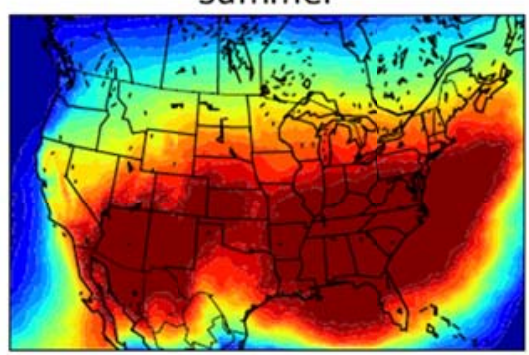

winter

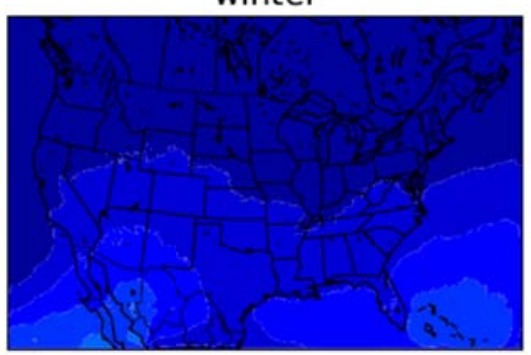

10

Figure S9: The difference in seasonal average daily maximum 8-hour $\mathrm{O}_{3}$ contribution from BCs inert and active $\mathrm{O}_{3}$ tracers, i.e., inert minus active, at layers between 240-750 mb. 

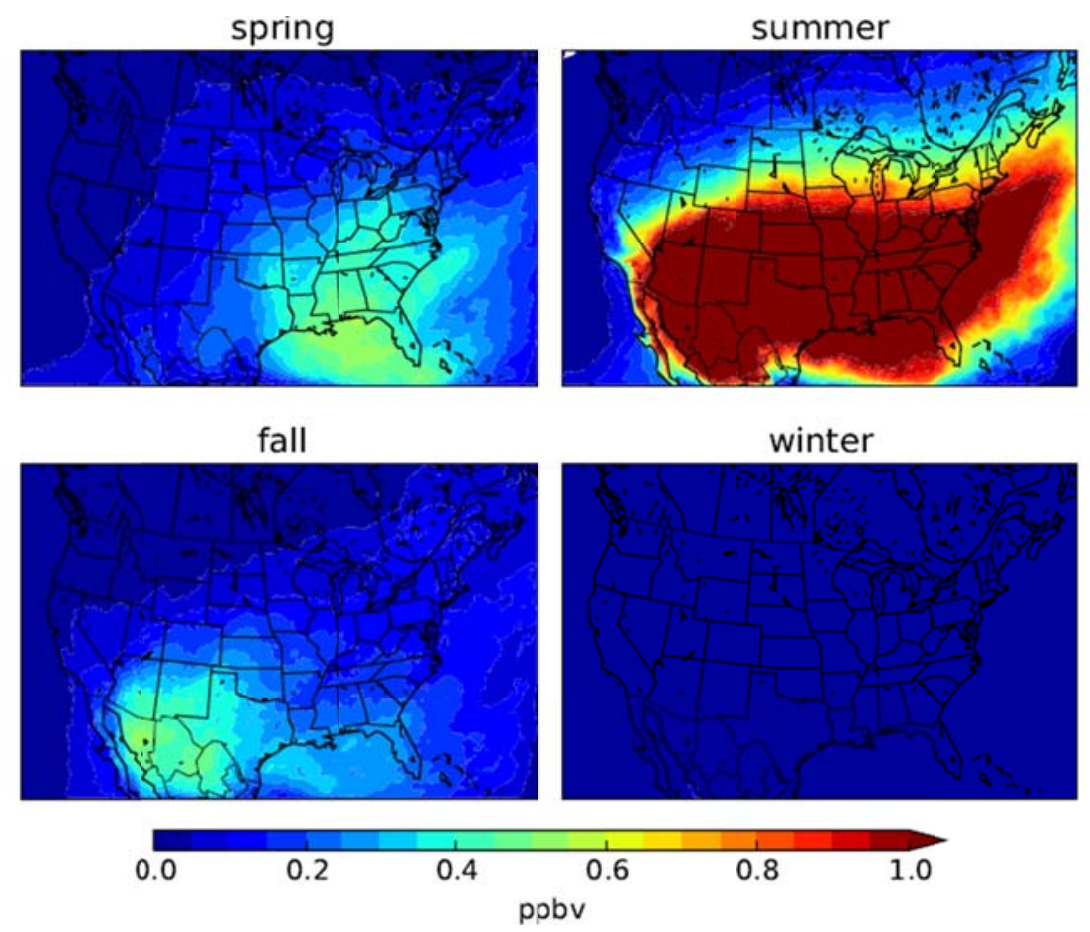

Figure S10: The difference in seasonal average daily maximum 8-hour $\mathrm{O}_{3}$ contribution from $\mathrm{BCs}$ inert and active $\mathrm{O}_{3}$ tracers, i.e., inert minus active, at layers above $240 \mathrm{mb}$. 